\title{
Semi-analytical Approach for Analyzing Vibro-Impact Systems
}

\author{
Algimantas Cepulkauskas ${ }^{1}$, Regina Kulvietienè ${ }^{1}$, Genadijus Kulvietis ${ }^{1}$, \\ and Jurate Mikucioniene ${ }^{2}$ \\ ${ }^{1}$ Vilnius Gediminas Technical University, Saulètekio 11, Vilnius 2040, Lithuania \\ \{algimantas_cepulkauskas, regina_kulvietiene, \\ genadijus_kulvietis\}@gama.vtu. It \\ ${ }^{2}$ Kaunas University of Technology, Kestucio 27, Kaunas 44025, Lithuania \\ jumik@ktu.It
}

\begin{abstract}
A semi-analytical approach, combining the features of analytical and numerical computations, is proposed. Separating the linear and nonlinear parts of equations of motion, the harmonic balance method and computer algebra have been synthesized for obtaining analytical solutions of the nonlinear part, whereas the linear part was solved by the numerical methods. On the basis of this technique, the numerical investigation of abrasive treatment process dynamics has been performed and regimes ensuring the most effective treatment process determined.
\end{abstract}

\section{Introduction}

Mechanical systems exhibiting impacts, so-called impact oscillators in the English literature at the present time, or vibro-impact systems in the Russian literature, are strongly nonlinear or piecewise linear, due to sudden changes in velocities of vibrating bodies at the instant of impact or friction forces when the velocity of motion changes its polarity. Their practical significance is considerable and the investigation of the motion of such systems was begun about fifty years ago [7].

Several methods of the theoretical analysis were developed and different models of impacts were presented [167]. The method of "fitting" which uses the Newton restitution coefficient seems to be most important. It is accurate and applicable under the assumption that the time during an impact is negligible. It can solve certain assumed periodic impact motion and its stability, but this procedure can be realized in the explicit form only for simple mechanical systems, simple types of periodic motion, and for undamped impactless motion.

As usual, the solution method must correlate with the type of motion equations and concurrently with the character of the initial mechanic system. The harmonic balance method was chosen for the investigation of a vibratory abrasive treatment process $[28$ as it is easily applied to systems where calculations are made by computer algebra methods and, in this case, considerably less computer memory is needed than by other methods [3]. As a result, we obtain a nonlinear algebra equation system with many very short expressions. So it is possible 
and expedient to process the results by numerical methods. The analytic calculation system VIBRAN is extremely effective in this case. Since an adequate method for solving the motion equation of dynamically complicated systems has to contain a large amount of analytic as well as numerical calculations, there must be a strong program connection among them. The analytic calculation system VIBRAN was selected in order to ensure this connection 3. The system is designed to generate subroutines in FORTRAN, to select and reject excessive operations while generating programs in accordance with analytic expressions. Besides, this system stands out by flexibility of input-output and the unique means for operations with sparse matrices.

\section{Implementation of Computer Algebra}

Consider the system of s degrees of freedom with the Lagrangian function [5]:

$$
L=L\left(q_{i}, \dot{q}_{i}, t\right) \quad(i=1,2, \ldots s),
$$

where $L$ is the Lagrangian function, $q_{i}, \dot{q}_{i}, t$ are generalized coordinates and velocities of the system and time; $s$ is the number of degrees of freedom.

The equations of motion of such a system are [4]:

$$
\frac{d}{d t}\left(\frac{\partial L}{\partial \dot{q}_{i}}\right)-\frac{\partial L}{\partial q_{i}}=F_{q i}
$$

These equations can be divided into the linear and nonlinear parts by formal replacements $L=L_{L}+L_{N}$ and $F_{q i}=F_{L i}+F_{N i}$. The equations of motion may now be expressed in the form:

$$
\left(\frac{d}{d t}\left(\frac{\partial L_{L}}{\partial \dot{q}_{i}}\right)-\frac{\partial L_{L}}{\partial q_{i}}-F_{L i}\right)+\left(\frac{d}{d t}\left(\frac{\partial L_{N}}{\partial \dot{q}_{i}}\right)-\frac{\partial L_{N}}{\partial q_{i}}-F_{N i}\right)=0,
$$

where $F_{q i}, F_{N i}, F_{L i}$ are generalized force, nonlinear (polynomial with respect to generalized coordinates and periodical or Fourier expansion of time) and linear parts; and $L_{N}, L_{L}$ are nonlinear and linear parts of the Lagrangian function, respectively.

The linear part can be formalized for numerical analysis without difficulty and we used special VIBRAN programs to analyze the nonlinear part of the system. The proposed method provides shorter expressions for analytical computation and allows the analysis of systems of higher order. After some well-known perturbations, the equations of motion can be rewritten in the matrix form:

$$
[M]\{\ddot{q}\}+[B]\{\dot{q}\}+[C]\{q\}=\{H(q, \dot{q}, t)\}+\{f(t)\},
$$

where $f(t)$ is the periodic function; $H(q, \dot{q}, t)$ is the nonlinear part of a system, calculated by a special VIBRAN program.

Solution of the abovementioned system can be expressed using the harmonic balance method in the form [314]:

$$
\{q\}=\left\{A_{0}\right\}+\left\{A_{1}\right\} \cos (\omega t)+\left\{A_{2}\right\} \sin (\omega t)+\ldots,
$$


where $\left\{A_{i}\right\}$ are the unknown vectors that can be found by the nonlinear algebraic equations.

According to the harmonic balance method, these equations for the first three vector coefficients in the matrix form are:

$$
[U]\{A\}-\{f\}-\{H(A)\}=\{0\},
$$

where $f_{i}$ are coefficients of Fourier expansion of the function $f(t)$. Analogously, equations for other harmonics could be found by the VIBRAN program. The expressions of $H_{i}$ and their derivatives required are expressed in closed form using computer algebra techniques by the FORTRAN code generation procedure. Special modifications were made to the terms with dry friction, and the integration procedure was developed by the Malkin method [7].

\section{Application of Abrasive Treatment Process Dynamics in the Investigation}

The new method for the treatment of miniature ring-shaped details was proposed in [8, where the internal surface of the detail is treated as well as an external one. During the vibratory treatment process the working medium particles are constantly striking each other. As a result, slight scratches and crater-shaped crevasses occur on the surface of treated details that form the surface microrelief. In this way, abrasive friction and impacts on the treated details perform the treatment process.

The equations of motion describe the dynamics of a vibratory abrasive treatment process [5]:

$$
\left\{\begin{array}{l}
m_{1} \ddot{x}_{1}+b \dot{x}_{1}+b_{k}\left(\dot{x}_{1}-\dot{x}_{a}\right)+\left(c+c_{1}\right) x_{1} \\
\quad=c \xi(t)-F_{m}\left(x_{1}\right)-F_{1} \operatorname{sign}\left(\dot{x}_{1}-\dot{x}_{a}\right)+b \dot{\xi}(t), \\
m_{a} \ddot{x}_{a}+b \dot{x}_{a}+b_{k}\left(\dot{x}_{a}-\dot{x}_{1}\right)+c x_{a}=c \xi(t)+F_{2} \operatorname{sign}\left(\dot{x}_{1}-\dot{x}_{a}\right)+b \dot{\xi}(t) .
\end{array}\right.
$$

where $m_{1}$ is the mass of components treated; $m_{a}$ is the mass of abrasive particles; the load mechanical properties are evaluated by elasticity $c$ and working medium resistance $b ; F_{1}, F_{2}$ are forces of dry friction between a component and abrasive; $b_{k}$ is viscosity resistance; the elasticity of magnetic field is evaluated by a stiffness coefficient $c_{1}$, the detail was additionally excited by generating a variable component of magnetic field $F_{m}\left(x_{1}\right)$. Its stiffness properties were obtained experimentally by the least squares method.

The kinematics excitation of a loaded vessel is $\xi(t)=A \sin \omega t$.

Analytic expressions obtained according to the VIBRAN program conclude the part of analytic calculations of $H(A)$ [3]. The corresponding derivatives are very simple and there are only 25 nonzero terms.

All the properties complying with the dynamic pattern of the process are investigated in the numerical way and the program itself is composed in the FORTRAN language. For this reason, in order to calculate the factors of analytic expressions and their partial derivatives, two FORTRAN subroutines have 
been generated: one for compiling a dictionary and another for calculating the expressions themselves. Besides, the program, created by applying the harmonic balance method for systems of differential equations in addition to the equations where amplitudes and constant components are found, also presents equation derivatives from unknown quantities. In this case, one or some criteria of further numerical parameter optimization may be calculated.

\section{Conclusions}

On the basis of solving nonlinear differential equations by a harmonic balance method and synthesis of the analytic calculation system VIBRAN, the investigation method of nonlinear systems with a dry friction effect has been created. This method combines the advantages of analytic calculation methods and computer algebra. They are a compound of the principle of parallel analytic-numerical calculation, where analytic rearrangements are applied only to the nonlinear part of the system, while concurrently the linear part of the system could be easily solved in the numerical way. The proposed method provides shorter expressions for analytic computation and allows the analysis of systems of higher order.

\section{References}

1. Baron, J.M.: Abrasive and Magnetic Treatment of Details and Cutters, St. Petersburg, Mashinostrojenie (in Russian), (1986)

2. Blekhman, I.I.: Forming the properties of nonlinear mechanical systems by means of vibration, Proc. IUTAM/IFToMM Symposium on Synthesis of Nonlinear Dynamical Systems, Solid Mechanics and Its Applications, vol. 73, Kluwer Academic Publ., Dordrecht (2000) 1-13.

3. Cepulkauskas, A., Kulvietiene, R., Kulvietis G.: Computer Algebra for Analyzing the Vibrations of Nonlinear Structures. Lecture Notes in Computer Science, Vol. 2657. Springer-Verlag, Berlin Heidelberg New York (2003) 747-753

4. Klymov, D.O., Rudenko, V.O.: Metody kompiuternoj algebry v zadachah mechaniki Nau, O(r)scow, (in russian), (1989)

5. Kulvietiene, R., Kulvietis, G., Fedaravicius, A., Mikucioniene, J.: Numeric-Symbolic Analysis of Abrasive Treatment Process Dynamics, Proc. Tenth World Congress of the Theory of Machines and Mechanisms, Vol. 6, Oulu, Finland, (1999) 2536-2541

6. Lewandowski, R.: Computational Formulation for periodic vibration of Geometrically Nonlinear Structures - Part 1: Theoretical Background. - Int. J. Solid Structures, 34 (15) (1997) 1925-1947

7. Malkin, I.G.: Some Problems of Nonlinear Oscillation Theory, Moscow, Gostisdat (in russian), (1956)

8. Mikucioniene, J.: Investigation of vibratory magnetic abrasive treatment process dynamics for miniature details, Ph.D. Thesis, KTU, Technologija, Kaunas, (1997) 